\title{
INTERPOLATION OF UNIFORMLY CONVEX BANACH SPACES
}

\author{
MICHAEL CWIKEL AND SHLOMO REISNER
}

\begin{abstract}
If $A_{0}$ and $A_{1}$ are a compatible couple of Banach spaces, one of which is uniformly convex, then the complex interpolation spaces $\left[A_{0}, A_{1}\right]_{\theta}$ are also uniformly convex for $0<\theta<1$. Estimates are given for the moduli of convexity and smoothness of $\left[A_{0}, A_{1}\right]_{\theta}$ in terms of these moduli for $A_{0}$ and $A_{1}$. In general, up to equivalence of moduli these estimates are best possible.
\end{abstract}

A result of Beauzamy [1, p. 71] states that if $A_{0}$ and $A_{1}$ are compatible Banach spaces, one of which is uniformly convex, then the spaces $S\left(p, \xi_{0}, A_{0}, \xi_{1}, A_{1}\right)$ obtained from $A_{0}$ and $A_{1}$ by the method of real interpolation, are also uniformly convex for $1<p<\infty$. In this note we present an analogue of this result for the complex interpolation method and give an estimate for the modulus of convexity of [ $\left.A_{0}, A_{1}\right]_{\theta}$ in terms of the moduli of $A_{0}$ and $A_{1}$. This estimate is best possible in general, up to equivalence of moduli. We remark that for the power type of the moduli, such an estimate is implicit in [10, section 1.9]. We use the notation and definitions of [7] and of [3,2] for concepts from the geometry of Banach spaces and from interpolation space theory respectively. The letters $A, A_{0}, A_{1}$ will always denote Banach spaces over the complex field. $\left(A_{0}, A_{1}\right)$ is a compatible couple (interpolation pair). The complex interpolation space $\left[A_{0}, A_{1}\right]_{\theta}$ is denoted by $A_{\theta}$.

We say that a positive function $f$ on $\mathbf{R}^{+}$is dominated by another function $g$ (notation: $f \prec g$ or $f(t) \prec g(t)$ ) if there are positive constants $a, b$ such that

$$
f(t) \leqslant a g(b t) \quad \text { for all } t \in \mathbf{R}^{+} .
$$

$f$ is equivalent to $g(f \sim g)$ if $f \prec g$ and $g \prec f$. We denote by $f^{-1}$ the inverse function of $f$ (if it exists). We denote the moduli of uniform convexity and uniform smoothness of $A$ by $\delta_{A}(\varepsilon)$ and $\rho_{A}(\tau)$ respectively.

It is known [4; 7, part II] that $\rho_{A}$ is an Orlicz function and that $\delta_{A}$ is equivalent to an Orlicz function $\tilde{\delta_{A}}\left(\delta_{A}(\varepsilon)\right.$ is defined only for $0 \leqslant \varepsilon \leqslant 2$ but $\tilde{\delta}_{A}$ is defined for all $\varepsilon \geqslant 0$ and equivalent to $\delta_{A}$ near 0 ). Also, $\rho_{A}(\tau)>0$ for $\tau>0$ (this can be concluded from the duality formula $\rho_{A}(\tau)=\frac{1}{2} \sup _{\varepsilon \geqslant 0}\left\{\tau \varepsilon-2 \delta_{A^{*}}(\varepsilon)\right\}$ and the fact that $\delta_{A^{*}}(\varepsilon) \prec$ $\varepsilon^{2}$ ) and being an Orlicz function, it turns out that $\rho_{A}$ is strictly increasing. $\tilde{\delta}_{A}$ is also strictly increasing if $A$ is uniformly convex ( $\delta_{A}$ itself is strictly increasing when $A$ is uniformly convex since $\delta_{A}(\varepsilon) / \varepsilon$ is nondecreasing). If $L$ is a Banach lattice of measurable functions on a measure space $(\Omega, \Sigma, \mu)$ we denote by $L(A)$ the space of

Received by the editors April 13, 1981 and, in revised form, June 29, 1981.

1980 Mathematics Subject Classification. Primary 46B20. 
$A$-valued Bochner measurable functions $f$ such that $\|f\|_{A} \in L$, normed by $\|f\|_{L(A)}$ $=\|\| f\left\|_{A}\right\|_{L}$. For $1 \leqslant p \leqslant \infty$ and $n \in \mathbf{N}$, we denote by $l_{p}^{n}$ or $L_{p}^{n}$ the space $\mathbf{R}^{n}$ with the norms $\left\|\left(a_{i}\right)\right\|=\left(\sum_{i=1}^{n}\left|a_{i}\right|^{p}\right)^{1 / p}$ or, respectively,

$$
\left\|\left(a_{i}\right)\right\|=\left(\frac{1}{n} \sum_{i=1}^{n}\left|a_{i}\right|^{p}\right)^{1 / p} .
$$

We conclude this introduction by recalling a construction of Calderón [3, 13.5]: If $M_{0}$ and $M_{1}$ are Banach lattices of functions on $(\Omega, \Sigma, \mu)$ and $0<\theta<1$ then the Banach lattice $M_{0}^{1-\theta} M_{1}^{\theta}$ is defined to be the space of all measurable functions $f$ which satisfy $|f| \leqslant \lambda\left|f_{0}\right|^{1-\theta}\left|f_{1}\right|^{\theta}$ for some $f_{0} \in M_{0}, f_{1} \in M_{1}, \lambda>0$ with $\left\|f_{i}\right\|_{M_{i}} \leqslant 1$, $i=0,1$. The norm in $M_{0}^{1-\theta} M_{1}^{\theta}$ is the infimum of the values of the above $\lambda$.

Finally, we wish to thank the referee of a previous version of this paper for helpful remarks and in particular, for calling our attention to Lemma 3 which enabled us to provide a simpler proof and obtain a stronger result.

TheOREM 1. Let $\left(A_{0}, A_{1}\right)$ be a compatible couple of Banach spaces and $0<\theta<1$.

(i) If at least one of $A_{0}, A_{1}\left(\right.$ e.g. $\left.A_{0}\right)$ is uniformly convex, then $A_{\theta}=\left[A_{0}, A_{1}\right]_{\theta}$ is uniformly convex and

$$
\delta_{A_{\theta}}(\varepsilon)>\delta_{A_{0}}\left(\varepsilon^{1 /(1-\theta)}\right)
$$

(also, $\delta_{A_{\theta}}(\varepsilon) \succ \delta_{A_{1}}\left(\varepsilon^{1 / \theta}\right)$ if $A_{1}$ is uniformly convex).

(ii) If both $A_{0}$ and $A_{1}$ are uniformly convex, then $\delta_{A_{\theta}}>\delta$ where $\delta$ is the inverse function of $\left(\delta_{A_{0}}^{-1}\right)^{1-\theta}\left(\delta_{A_{1}}^{-1}\right)^{\theta}$.

(iii) $\rho_{A_{\theta}} \prec \rho$, where $\rho$ is the inverse function of $\left(\rho_{A_{0}}^{-1}\right)^{1-\theta}\left(\rho_{A_{1}}^{-1}\right)^{\theta}$.

We remark that simple examples (e.g. $L_{p}$-spaces) show that the above estimates are, in general, best possible up to equivalence of moduli.

By duality between $\delta_{A}$ and $\rho_{A^{*}}, \delta_{A^{*}}$ and $\rho_{A}$ (up to equivalence of functions), (iii) follows from (i) and (ii). For the proof of (i) and (ii) we introduce the following definitions and lemmas.

For a strictly increasing Orlicz function $\phi$, we define the lattice norm \|\|$_{\phi}$ on $\mathbf{R}^{2}$ by

$$
\|(a, b)\|_{\phi}=\inf \{\rho>0|| a \mid / \rho+\phi(|b| / \rho) \leqslant 1\},
$$

which is the same as

$$
\|(a, b)\|_{\phi}=\inf \left\{\lambda>0|\exists(c, d),| c|+| d|\leqslant 1,| a|\leqslant \lambda| c|,| b \mid \leqslant \lambda \phi^{-1}(|d|)\right\} .
$$

We denote the Banach lattice $\left(\mathbf{R}^{2},\|\|_{\phi}\right)$ by $M_{\phi}$.

LEMMA 2. Let $\phi_{0}, \phi_{1}$ be two strictly increasing Orlicz functions and $\phi=$ $\left[\left(\phi_{0}^{-1}\right)^{1-\theta}\left(\phi_{1}^{-1}\right)^{\theta}\right]^{-1}$. Then

$$
\|\|_{\phi(\alpha \cdot)} \leqslant\|\|_{M_{\phi_{0}}^{1-\theta} M_{\phi_{1}}^{\theta}} \leqslant\|\|_{\phi}
$$

where $\alpha=\min (\theta, 1-\theta)$. 
Proof. It is clear that $\phi$ is an Orlicz function.

Since $\phi_{i}^{-1}$ are concave and $\phi_{i}^{-1}(0)=0(i=0,1)$, we have for $0<\lambda \leqslant 1$ and $t \geqslant 0$

$$
\lambda \phi_{i}^{-1}(t) \leqslant \phi_{i}^{-1}(\lambda t) .
$$

Consequently, if $0<t_{1} \leqslant t_{2}$ we have

$$
\phi_{i}^{-1}\left((1-\theta) t_{1}+\theta t_{2}\right) \geqslant \frac{(1-\theta) t_{1}+\theta t_{2}}{t_{2}} \phi_{i}^{-1}\left(t_{2}\right) \geqslant \theta \phi_{i}^{-1}\left(t_{2}\right) \geqslant \theta \phi_{i}^{-1}\left(t_{1}\right) .
$$

In a similar way, for $0<t_{2} \leqslant t_{1}$ we have

$$
\phi_{i}^{-1}\left((1-\theta) t_{1}+\theta t_{2}\right) \geqslant(1-\theta) \phi_{i}^{-1}\left(t_{1}\right) \geqslant(1-\theta) \phi_{i}^{-1}\left(t_{2}\right)
$$

hence for all $t_{1}, t_{2} \geqslant 0$

$$
\left[\phi_{0}^{-1}\left(t_{1}\right)\right]^{1-\theta}\left[\phi_{1}^{-1}\left(t_{2}\right)\right]^{\theta} \leqslant \alpha^{-1}\left[\phi_{0}^{-1}\left((1-\theta) t_{1}+\theta t_{2}\right)\right]^{1-\theta}\left[\phi_{1}^{-1}\left((1-\theta) t_{1}+\theta t_{2}\right)\right]^{\theta}
$$

with $\alpha=\min (\theta, 1-\theta)$.

Now suppose $\|(a, b)\|_{M_{\phi_{0}}^{1-\theta} M_{\phi_{1}}^{\theta}} \leqslant 1$. By the definition, there are $c_{1}, c_{2}, d_{1}, d_{2}$ with $\left|c_{j}\right|+\left|d_{j}\right| \leqslant 1, j=1,2$, such that

$$
|a| \leqslant\left|c_{1}\right|^{1-\theta}\left|c_{2}\right|^{\theta}
$$

and

$$
|b| \leqslant\left[\phi_{0}^{-1}\left(\left|d_{1}\right|\right)\right]^{1-\theta}\left[\phi_{1}^{-1}\left(\left|d_{2}\right|\right)\right]^{\theta}
$$

then

$$
|a| \leqslant(1-\theta)\left|c_{1}\right|+\theta\left|c_{2}\right|
$$

and

$$
|b| \leqslant \alpha^{-1}\left[\phi_{0}^{-1}\left((1-\theta)\left|d_{1}\right|+\theta\left|d_{2}\right|\right)\right]^{1-\theta}\left[\phi_{1}^{-1}\left((1-\theta)\left|d_{1}\right|+\theta\left|d_{2}\right|\right)\right]^{\theta}
$$

or

$$
\alpha|b| \leqslant \phi^{-1}\left((1-\theta)\left|d_{1}\right|+\theta\left|d_{2}\right|\right) .
$$

Since

$$
\left[(1-\theta)\left|c_{1}\right|+\theta\left|c_{2}\right|\right]+\left[(1-\theta)\left|d_{1}\right|+\theta\left|d_{2}\right|\right] \leqslant 1
$$

this implies that $\|(a, b)\|_{\phi(\alpha \cdot)} \leqslant 1$ thus proving the left-hand side of (1). The right-hand side of (1) is straightforward.

LEMMA 3. $\delta_{A}>\delta$ if and only if there are positive constants $a, b$ such that for all $x, y$ in $A$ holds

$$
\frac{\|x+y\|^{2}+\|x-y\|^{2}}{2} \leqslant 1 \Rightarrow\|x\|+a \delta(b\|y\|) \leqslant 1
$$

Proof. If (2) holds, then obviously $\delta_{A}>\delta$ (take $z, w$ with $\|z\|,\|w\| \leqslant 1$ and write $z=x+y, w=x-y)$.

On the other hand, by [4], ([5]) $\delta_{A} \sim \delta_{L_{2}(A)}$. Assume $\delta_{L_{2}(A)}>\delta$. Given that

$$
\frac{\|x+y\|^{2}+\|x-y\|^{2}}{2} \leqslant 1
$$


we consider $x$ as a constant function in $L_{2}(A)$ over a probability space and look at the monotone basic sequence of two elements $\{x, \varepsilon y\}$ in $L_{2}(A)$, where $\varepsilon$ is a Bernoulli variable. This sequence satisfies the requirement of Proposition 2.1 in [8] hence, by that proposition

$$
\|x\|+\delta_{L_{2}(A)}(\|y\|) \leqslant 1 .
$$

Proof of Theorem 1. We assume, as we may, that $\boldsymbol{\delta}_{A_{i}}$ are Orlicz functions, strictly increasing if $A_{i}$ is uniformly convex.

We prove first (ii). By Lemma 3, (2) is satisfied by $A_{i}, \delta_{A_{i}}$ with constants $a_{i}, b_{i}$ $(i=0,1)$. If $\phi_{i}=a_{i} \delta_{A_{i}}\left(b_{i}\right)$ then this means that the operator

$$
T: A_{i} \times A_{i} \rightarrow A_{i} \times A_{i}
$$

defined by $T(z, w)=((z+w) / 2,(z-w) / 2)$, has norm $\leqslant 1$ as an operator

$$
T: L_{2}^{2}\left(A_{i}\right) \rightarrow M_{\phi_{i}}\left(A_{i}\right)
$$

for both $i$ 's.

Hence $T$ has norm $\leqslant 1$ as an operator

$$
T: L_{2}^{2}\left(A_{\theta}\right) \rightarrow\left[M_{\phi_{0}}\left(A_{0}\right), M_{\phi_{1}}\left(A_{1}\right)\right]_{\theta} .
$$

The lattice $M_{\phi_{0}}^{1-\theta} M_{\phi_{1}}^{\theta}$ clearly has the convergence property required for the result in paragraph (i) of $[3,13.6]$ and that result yields that

$$
\left[M_{\phi_{0}}\left(A_{0}\right), M_{\phi_{1}}\left(A_{1}\right)\right]_{\theta}=M_{\phi_{0}}^{1-\theta} M_{\phi_{1}}^{\theta}\left(A_{\theta}\right)
$$

which, together with Lemma 2 yields that $T$ has norm $\leqslant 1$ as an operator

$$
T: L_{2}^{2}\left(A_{\theta}\right) \rightarrow M_{\phi(\alpha \cdot)}\left(A_{\theta}\right)
$$

where $\phi=\left[\left(\phi_{0}^{-1}\right)^{1-\theta}\left(\phi_{1}^{-1}\right)^{\theta}\right]^{-1}$.

The last assertion is just (2) for $A=A_{\theta}, \delta=\phi, a=1, b=\alpha$ from which (ii) follows easily.

The proof of (i) goes along the same lines, we use (2) for $A_{0}, \delta_{A_{0}}, a_{0}, b_{0}$ and take into account that in every Banach space we have

$$
\left(\frac{\|x+y\|^{2}+\|x-y\|^{2}}{2}\right)^{1 / 2} \geqslant \max (\|x\|,\|y\|)
$$

i.e. $T: L_{2}^{2}\left(A_{1}\right) \rightarrow l_{\infty}^{2}\left(A_{1}\right)$ has norm $\leqslant 1$.

Hence $T: L_{2}^{2}\left(A_{\theta}\right) \rightarrow\left[M_{\phi_{0}}\left(A_{0}\right), l_{\infty}^{2}\left(A_{1}\right)\right]_{\theta}$ has norm $\leqslant 1$; this yields for $x, y \in A_{\theta}$

$$
\frac{\|x+y\|^{2}+\|x-y\|^{2}}{2} \leqslant 1 \Rightarrow\|x\|^{1 /(1-\theta)}+\phi_{0}\left(\|y\|^{1 /(1-\theta)}\right) \leqslant 1
$$

hence, if $z, w \in A_{\theta},\|z\|,\|w\| \leqslant 1$ then

$$
\begin{aligned}
\left\|\frac{z+w}{2}\right\| & \leqslant\left[1-\phi_{0}\left(\left\|\frac{z-w}{2}\right\|^{1 /(1-\theta)}\right)\right]^{1-\theta} \\
& \leqslant 1-(1-\theta) \phi_{0}\left(\left\|\frac{z-w}{2}\right\|^{1 /(1-\theta)}\right)
\end{aligned}
$$

which proves (i). 
REMARK. Another approach which can be used to prove similar results proceeds via use of the martingale characterization of uniform convexity of [8 and 6]. A disadvantage of this approach is that in the general case it yields only isomorphic results. However, for functions $\delta(\varepsilon)$ of the form $\varepsilon^{q}$ it gives the same result as here, and in this case it works also for the real interpolation method. Namely, if $A_{i}$ are isomorphic to spaces with moduli of uniform convexity $>\varepsilon^{q_{i}}$, then $\left(A_{0}, A_{1}\right)_{\theta, s}$ is isomorphic to a space with modulus of convexity $>\varepsilon^{s}$ for every $s$ with $q_{\theta} \leqslant s<\infty$ $\left(1 / q_{\theta}=(1-\theta) / q_{0}+\theta / q_{1}\right)$.

\section{REFERENCES}

1. B. Beauzamy, Espaces d'interpolation réels: Topologie et géométrie, Lecture Notes in Math., vol. 666, Springer-Verlag, Berlin and New York, 1978.

2. J. Bergh and J. Löfström, Interpolation spaces. An introduction, Grundlehren der Math. Wissenschaften, Bd. 223, Springer-Verlag, Berlin and New York, 1976.

3. A. P. Calderón, Intermediate spaces and interpolation, the complex method, Studia Math. 24 (1964), $113-190$.

4. T. Figiel, On the moduli of convexity and smoothness, Studia Math. 56 (1976), 121-155.

5. T. Figiel and G. Pisier, Séries aléatoires dans les espaces uniformément convexes ou uniformément lisses, C. R. Acad. Sci. Paris Ser. A 279 (1974), 611-614.

6. D. J. Garling, Convexity, smoothness and martingale inequalities, Israel J. Math. 29 (1978), 189-198.

7. J. Lindenstrauss and L. Tzafriri, Classical Banach spaces, Parts I, II, Ergebnisse der Math. und ihrer Grenzgebiete, Bds. 92, 97, Springer-Verlag, Berlin and New York, 1977, 1979.

8. G. Pisier, Martingales with values in uniformly convex spaces, Israel J. Math. 20 (1975), 326-350.

9. Some applications of the complex interpolation method to Banach lattices, J. Analyse Math. 35 (1979), 264-280.

10. La méthode d'interpolation complexe: Application aux treillis de Banach, École Polytechnique Séminaire d'Analyse Fonctionelle, 1978-79, Exp. 17.

Department of Mathematics, Technion-IsRael Institute of Technology, Haifa 32000, IsRael

Department of Mathematics, Texas A \& M University, College Station, Texas 77843 